\title{
Early Changes in Bone Specific Turnover Markers During the Healing Process After Vertebral Fracture
}

\author{
Hiroyuki Hashidate ${ }^{*}$, , Mikio Kamimura $^{2}$, Hiroyuki Nakagawa ${ }^{3}$, Kenji Takahara ${ }^{4}$, Shota Ikegami ${ }^{1}$, \\ Shigeharu Uchiyama ${ }^{1}$ and Hiroyuki Kato ${ }^{1}$
}

${ }^{I}$ Department of Orthopedic Surgery, Shinshu University, Japan

${ }^{2}$ Center of Osteoporosis and Spinal Disorders: Kamimura Orthopaedic Clinic, Japan

${ }^{3}$ Department of Orthopedic Surgery, Suwa Red Cross Hospital, Japan

${ }^{4}$ Department of Orthopedic Surgery, Ina Central Hospital, Japan

\begin{abstract}
Background: The present study measured longitudinal changes in bone turnover markers in elderly patients with vertebral fracture and investigated the relationship among bone turnover markers, duration of bed rest and bone mineral density (BMD).

Methods: Criteria for patient selection were 50 years in age and older, and presence of VF. Serum bone-specific alkaline phosphatase (BAP) was measured as a marker of bone formation. Urinary crosslinked N-terminal telopeptides of type I collagen (NTX) was measured as a marker of bone resorption. In principle, samples were collected just after injury, within $24 \mathrm{~h}$, and 1, 2, 3, 5 and 8 weeks after. We also measured duration of bed rest and BMD.

Results: The study population consisted of 42 cases. The average BMD of the lumbar vertebrae was $0.670 \pm 0.174 \mathrm{~g} / \mathrm{cm} 2$. Bed rest period was $17.9 \pm 8.8$ days. BAP showed significantly higher values at 2 and 3 weeks compared with the baseline value. Thereafter, BAP progressively decreased until 8 weeks. Urinary NTX was increased soon after the onset of pain with the same patterns in BAP. Urinary NTX values reached a peak at 3 weeks, and then they kept significantly higher values until 8 weeks. The peak value of serum BAP was affected by the duration of bed rest, although that of the urinary NTX was not. The peak values of serum BAP and urinary NTX showed negative correlations with the initial BMD values.

Conclusions: Bone turnover markers remained higher at 8 weeks, even patients symptom was healed after VF. Bone turnover markers were affected on physical activity and BMD.
\end{abstract}

Keywords: Vertebral fracture, bone turnover marker, osteoporosis.

Osteoporosis is a systemic skeletal disease characterized by low bone mass and microarchitectural deterioration of bone tissue, with a consequent increase in bone fragility and susceptibility to fracture [1]. Vertebral fractures (VF) are the classic hallmark of osteoporosis [2].

Generally, it is thought that bone loss accelerates with high turnover of the bone after menopause. This accelerated turnover declines over time until a steady state of low turnover is eventually reached. Further, some studies have indicated that there is high turnover in the early postmenopausal period that decreases through the seventh and eighth decade, over 15 years after menopause [3, 4]. Recently, we reported that bone turnover markers associated with back pain (BP) are significantly increased in elderly women, and speculated that these bone turnover markers are increased by unrecognized VF in patients with BP [5].

Several clinical studies have pointed out that bone turnover markers after fractures increase with fracture

*Address correspondence to this author at the Department of Orthopaedic Surgery, Shinshu University, Asahi 3-1-1 Matsumoto, Nagano, Japan; Tel: +81-263-37-2659; Fax: +81-263-35-8844;

E-mail: hashidate4789@yahoo.co.jp healing [6-15]. Usually, VF requires prolonged bed rest for pain relief. Bone resorption markers are strongly related to physical activity [16-20]. Therefore, VF or low physical activity such as bed rest might differently influence bone turnover markers. However, there have been no reports regarding the relationship between bed rest after VF and bone turnover markers. Longitudinal and prospective studies are desirable to elucidate the time course of changes in bone turnover markers in elderly women and the precise relationships between bone turnover markers, BP, and VF.

In our institute, elderly patients with severe BP were evaluated using radiograph (X-P), magnetic resonance imaging (MRI) and bone scintigram (BS). Many of these patients were diagnosed as having VF and were treated with bed rest [21]. The present study monitored longitudinal changes in bone turnover markers in elderly patients with VF and investigated the relationship among bone turnover markers, duration of bed rest, and bone mineral density (BMD).

\section{MATERIALS AND METHODOLOGY}

\section{Patients}

Criteria for patient selection were 50 years of age and older and the presence of osteoporotic VF with or without a 
history of minor trauma. Patients with high-energy injuries were excluded from this study. Forty-two consecutive patients with VF combined with osteoporosis were admitted to our institution because of severe pain when changing positions and inability to sit up. All the patients gave informed consent for examination and medical treatment. This study was carried in accordance with the Declaration of Helsinki. The study was approved by the Ethics Committee of Suwa Red Cross Hospital. The authors report no conflicts of interest. The authors alone are responsible for the content and writing of the paper.

\section{Radiologic Evaluation}

Patients underwent a series of imaging studies including anterior-posterior (AP) and lateral X-P, MRI of the spine, and BS within one week after admission.

Diagnosis of VF on MRI was made when T1-weighted images showed low intensity images in the involved vertebra on the sagittal plane and intense radiotracer uptake on BS. In addition, BMD of the AP lumbar spine was measured using dual X-ray absorptiometry (DXA) (QDR2000 Hologic, Waltham, MA, USA) for unaffected vertebra.

The final diagnosis of VF was based on radiographic findings on MRI and BS, and the clinical course of the patient.

\section{Treatment of Osteoporotic VF}

Patients were treated conservatively with bed rest until severe BP had diminished and patients could sit up with mild, tolerable BP. The duration of bed rest was defined as the period from the onset of VF to the time patients could ambulate with external fixation by orthosis or plaster cast. During this period, the patients were allowed to raise in bed if doing so without pain. Patients who could sit up with no pain were allowed to walk with external fixation by orthosis or plaster cast.

\section{Biochemical Markers}

Serum bone-specific alkaline phosphatase (BAP) was measured as a marker of bone formation. Urinary level of cross-linked N-terminal telopeptides of type I collagen (NTX) was measured as a marker of bone resorption by enzyme-linked immunosorbent assay (ELISA). In principle, samples of venous blood and spot urine were collected six times as closely as possible in accordance with the following schedule: just after, within $24 \mathrm{~h}$, and 1, 2, 3, 5, and 8 weeks after injury. Patients were encouraged to undergo measurements more than four times. Second spot urine samples were collected in the morning, to avoid first morning urine. The samples were stored at $-20^{\circ} \mathrm{C}$ until analysis. The immunoassay was performed by SRL Inc. (Tokyo, Japan).

\section{Statistical Analysis}

The results are expressed as the means and standard deviation (SD). Differences in the levels of bone turnover markers between at the onset of BP and at each examination point were assessed using Student's unpaired t-test. Significance was accepted at a p-value of less than 0.05 . The correlations between bone turnover markers and period of bed rest or BMD were assessed using Pearson's correlation coefficient.

\section{RESULTS}

The study population consisted of 42 cases, 28 women and 14 men, ranging in age from 57 to 95 years (mean, 77.7 years). The average BMD of the lumbar vertebrae was 0.670 $\pm 0.174 \mathrm{~g} / \mathrm{cm}^{2}$ (Table 1). Bed rest period was $17.9 \pm 8.8$ days for patients with VF. All patients showed a low T1-weighted signal of the affected vertebra on MRI and intense radiotracer uptake on BS. All patients demonstrated a decrease in BP with conservative treatment.

Table 1. Characteristics of patients

\begin{tabular}{|c|rl|}
\hline Characteristics & Number or Mean \pm SD (Range) \\
\hline \hline Male sex (Female) & $14(28)$ \\
\hline Age (years) & $77.7 \pm 8.2$ & $(57-95)$ \\
\hline Weight $(\mathrm{kg})$ & $51.0 \pm 9.7$ & $(35-75)$ \\
\hline Height $(\mathrm{cm})$ & $154.7 \pm 7.3$ & $(140-173)$ \\
\hline Lumbar spine BMD & $0.670 \pm 0.174$ & $(1.073-0.409)$ \\
\hline
\end{tabular}

\section{The Time Course of Serum BAP Value (Fig. 1)}

BAP was $31.8 \pm 14.3 \mathrm{U} / 1$ at the baseline, which quickly increased to reach $45.4 \pm 14.1 \mathrm{U} / 1$ at 3 weeks after the onset of BP, showing significantly higher values at 2 and 3 weeks compared with the baseline value. Thereafter, BAP decreased to $38.3 \pm 15.2 \mathrm{U} / \mathrm{l}$ at 8 weeks. Although the difference was not significant compared to the baseline value, BAP continued to show slightly higher values until the 8th week.

\section{The Time Course of Urinary NTX Value (Fig. 2)}

Urinary NTX was increased soon after the onset of BP, with the same patterns as for BAP. At 2 weeks after the onset of BP, urinary NTX rose from $73.2 \pm 52.4$ to $131.3 \pm$ $77.9 \mathrm{nmol} \mathrm{BCE} / \mathrm{mmol}$ Cr. Urinary NTX reached a peak at 3 weeks, and then maintained significantly higher values compared with the baseline until the 8th week. Both serum BAP and urinary NTX reached peak values 3 weeks after trauma, suggesting bone formation and resorption increase after fracture.

\section{Effects of BMD and Bed Rest Period on Biochemical Markers}

In these patients, the relation between the peak values of bone turnover markers, the duration of bed rest, and BMD were examined. There was no correlation between BMD and bed rest period. The peak value of BAP, however, had weak correlation with the duration of bed rest (Fig. 3): as the rest period became longer, the BAP value became significantly lower. In contrast, the peak value of urinary NTX did not correlate with the duration of bed rest (Fig. 4).

The peak values of both serum BAP and urinary NTX showed negative correlation with the BMD value at the onset of BP (Figs. 5, 6). 


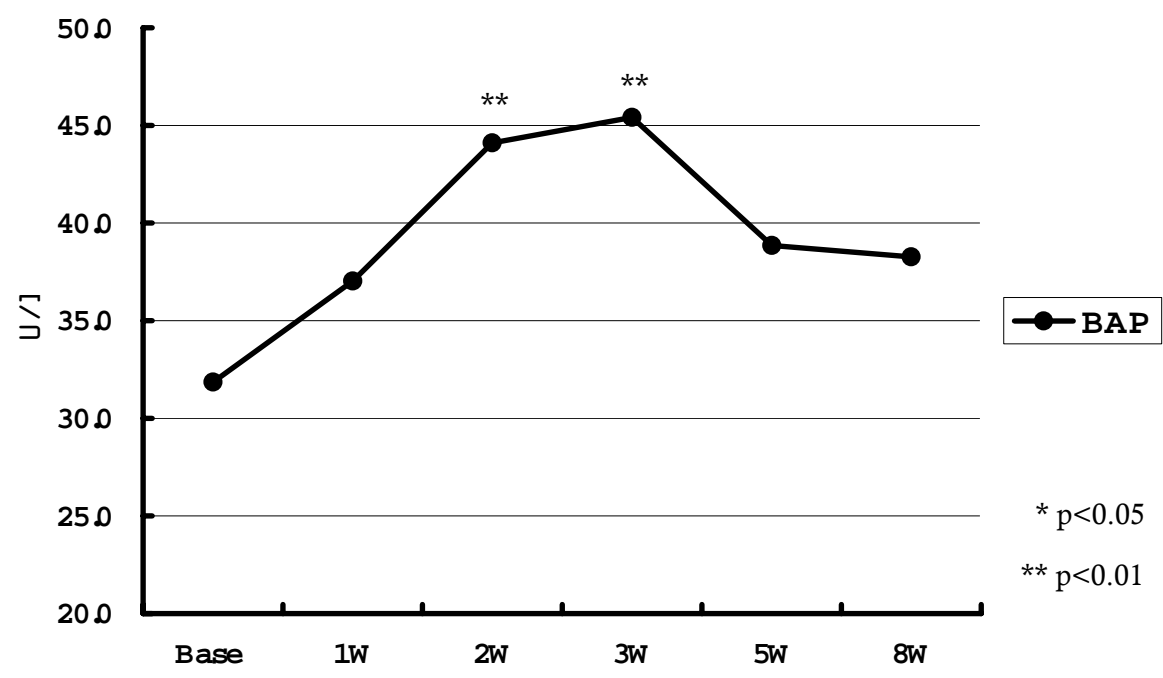

\begin{tabular}{c|c|c|c|c|c|c}
\hline & Base line & $1 \mathrm{~W}$ & $2 \mathrm{~W}$ & $3 \mathrm{~W}$ & $5 \mathrm{~W}$ & $8 \mathrm{~W}$ \\
\hline BAP & $31.8 \pm 14.3$ & $37.0 \pm 18.2$ & $44.1 \pm 12.3$ & $45.4 \pm 14.1$ & $38.9 \pm 15.6$ & $38.3 \pm 15.2$ \\
\hline No. & 34 & 23 & 20 & 32 & 30 & 24 \\
\hline
\end{tabular}

Fig. (1). Baseline values and longitudinal absolute mean value $( \pm S D)$ of serum BAP.

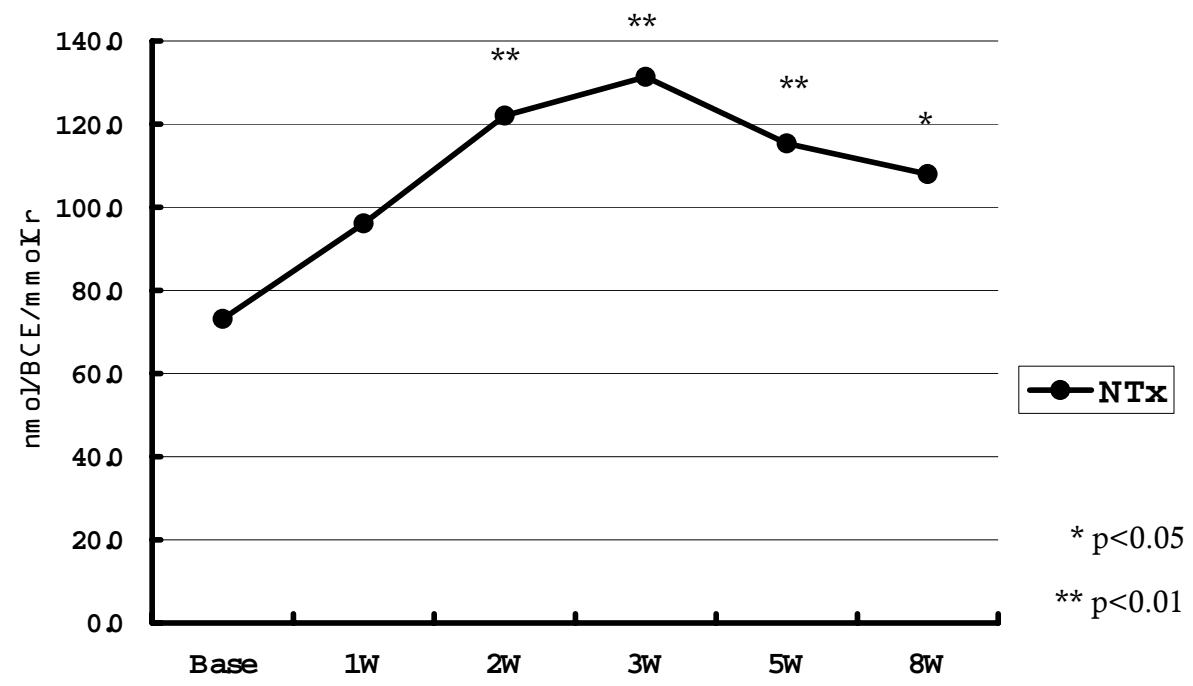

\begin{tabular}{c|c|c|c|c|c|c}
\hline & Base line & $1 \mathrm{~W}$ & $2 \mathrm{~W}$ & $3 \mathrm{~W}$ & $5 \mathrm{~W}$ & $8 \mathrm{~W}$ \\
\hline NTX & $73.2 \pm 52.4$ & $96.1 \pm 52.7$ & $122.0 \pm 67.0$ & $131.3 \pm 77.9$ & $115.3 \pm 59.4$ & $108.0 \pm 62.1$ \\
\hline No. & 35 & 23 & 19 & 32 & 30 & 23 \\
\hline
\end{tabular}

Fig. (2). Baseline values and longitudinal absolute mean value $( \pm \mathrm{SD})$ of urinary NTX.

\section{DISCUSSION}

VF is a common osteoporotic fracture in elderly patients, and the incidence of VF has increased in recent years. In patients with osteoporosis, VF frequently causes BP [21-23]. As BP usually ceases within a few weeks, these fractures are not considered to induce severe clinical problems in most cases [24]. Furthermore, Vogt et al. reported that the fractures are recognized and diagnosed in only one third of patients with VF [25]. However, regardless of the presence of trauma, severe BP in patients with osteoporosis tended to indicate the presence of VF [21]. In patients with osteoporosis, VF causing severe BP requires a prolonged bed rest period for pain relief regardless of traumatic episode 
and/or vertebral deformity. Osteoporotic VF demonstrates various interesting clinical features, and such fractures may variously influence bone turnover markers.

Various biochemical markers reflect bone metabolism. When bone resorption occurs, breakdown products of type I collagen are released into the circulatory system. NTX is one of these breakdown products and the urinary NTX level has been reported to be a specific and sensitive marker of bone resorption [26]. BAP is a specific product of osteoblasts and is used as a typical bone formation marker to evaluate bone turnover. In the present study it was shown that both bone formation and resorption markers increased over 8 weeks after fracture.
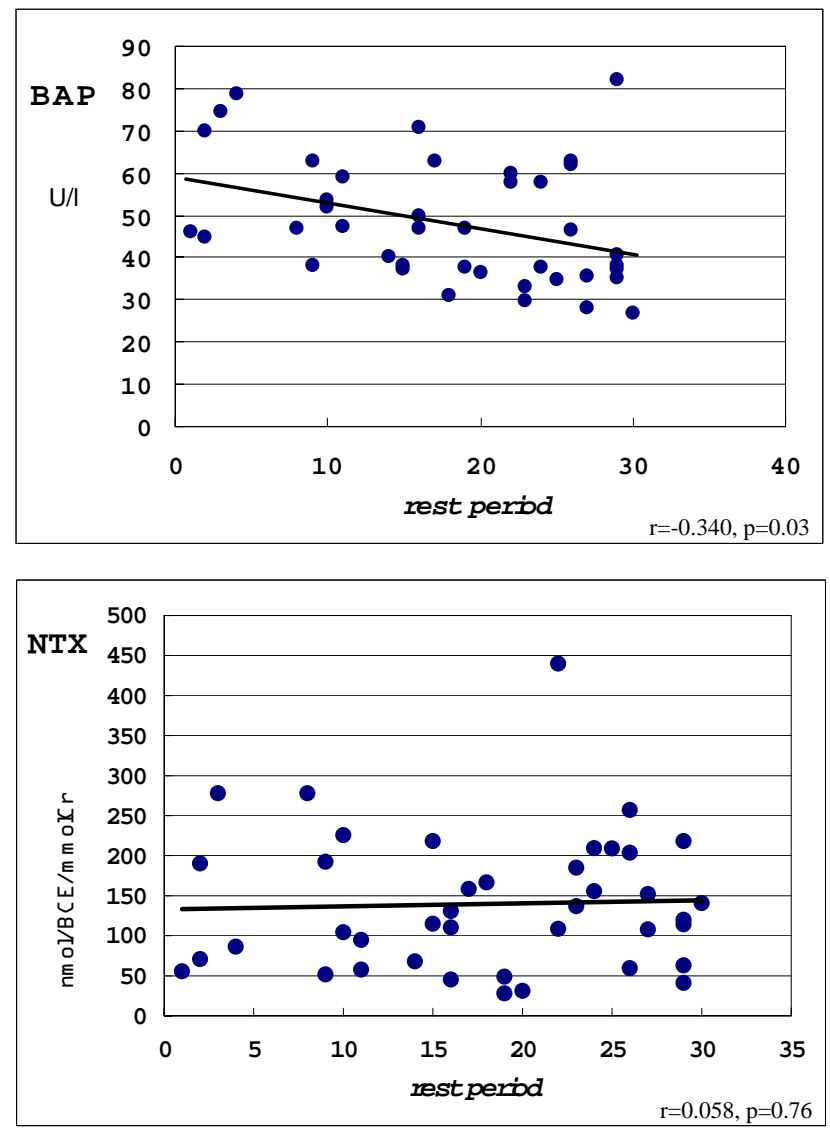

Figs. (3, 4). It is not correlation between NTX, but correlation between NTX and duration of bed rest.

Many authors have reported that bone turnover markers increased after fractures [6-15]. For example, hip fracture is one of the most common fractures in osteoporotic patients, comprising mainly femoral trochanter and neck fracture. Despite differences in post-operative therapy, the change of bone turnover markers during the healing of these fractures showed a similar pattern [13, 27]. However, the increase of bone turnover markers was significantly greater in trochanter fracture than in neck fracture, indicating that each type of fracture showed a different amount of influence on bone turnover markers.

In this study, patients with osteoporotic VF showed a tendency toward a negative correlation between bone turnover markers and BMD. It was suspected that mechanical force caused a larger area of vertebral body destruction for the patients with lower BMD, resulting in stronger effect on the bone turnover markers.

Generally, it is considered that the fracture may have a direct impact on bone formation and immobilization following fracture may induce an increase in bone resorption [7]. According to the previous studies, bone resorption markers are strongly related to physical activity [16-20]. In fact, some authors have found that bone resorption markers were increased [16-20] while bone formation markers decreased with bed rest [17]. Therefore, decreased physical activity such as bed rest usually causes an increase in bone resorption and an inhibition of bone formation. However, there are no reports describing changes in bone turnover marker due to osteoporotic VF with consideration for the duration of bed rest.
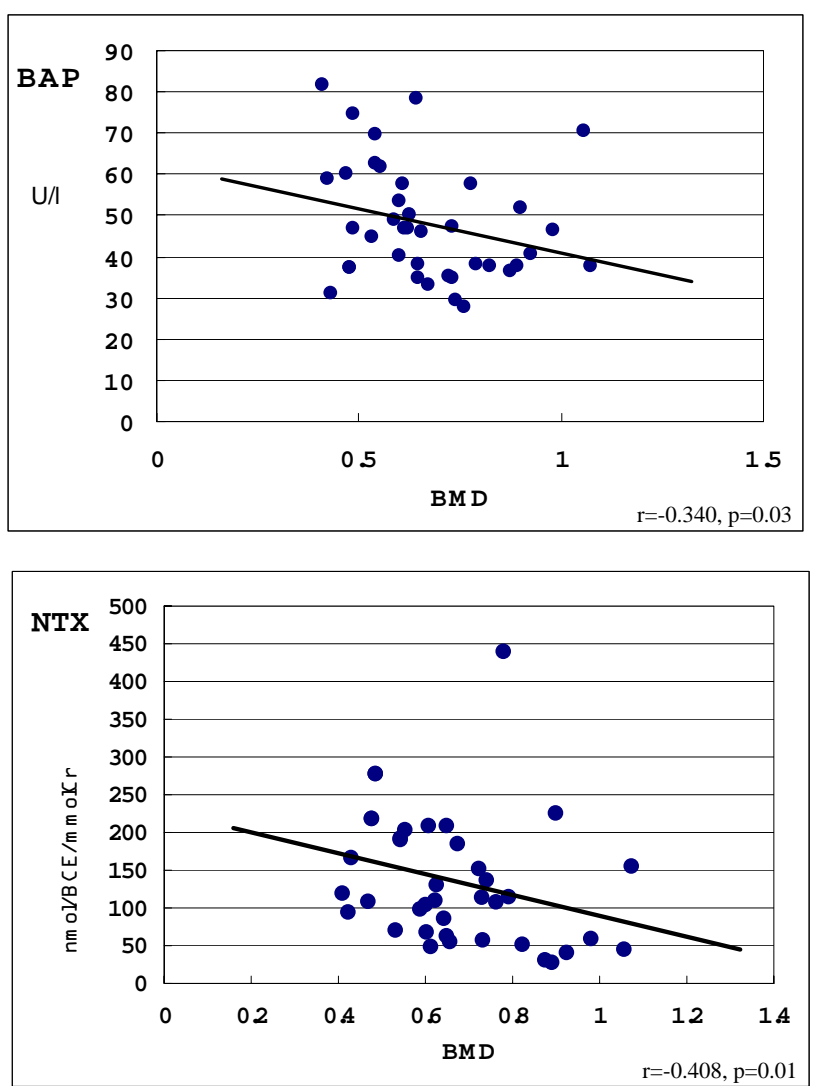

Figs. $(5,6)$. There was a correlation between BAP and BMD, as well as a correlation between NTX and BMD.

The present study demonstrated that the peak value of bone formation marker correlated with the duration of bed rest, while the peak value of bone resorption marker did not. It was considered that these correlations of bone formation marker were influenced by mechanical load to VF or physical activity. During fracture healing, acceleration of bone fusion might increase both bone formation and resorption markers. However, long-term bed rest accelerated bone resorption and decreased bone formation. For these reasons, it was speculated that early mobilization of patients increases mechanical load of VF before healing, accelerating the fracture healing process and increasing bone formation. Another possible reason is that long-term bed rest induces greater decline in bone formation; however it must be emphasized that the level is not significant, and further research is required. 
This study has some limitations. First, the study was not population-based and the sample size was not large enough to draw definite conclusions. Second, not all data was collected in each period, and therefore time course of bone turnover markers might not be accurately reflected.

Recently, many studies have shown that an increase in bone turnover after menopause persists for longer than was previously believed. An increased incidence of fractures with aging has been demonstrated $[28,29]$. Women aged over 80 years frequently have VF and the presence of such fractures is a strong risk factor for subsequent fractures [21, 25, 30, 31]. We previously reported that NTX and BAP levels were increased significantly in women over 80 years compared to those at age 60 , and specifically in patients with BP associated with unrecognized fracture [5]. Considering these findings, elderly women with osteoporosis might show repeated VF regardless of symptoms such as BP, and VF might obviously affect the level of bone turnover markers. Therefore, in elderly patients with osteoporosis, bone turnover markers may be unstable.

\section{CONCLUSION}

Bone turnover markers remained higher at 8 weeks, even in patients for whom symptoms had ceased after VF. Bone turnover markers were likely to be affected by physical activity and BMD. An increase in bone turnover markers after vertebral fracture and the subsequent decrease may reflect bone formation and remodeling during the fracture healing process. High turnover may reflect not only bone fragility but also fracture healing.

\section{REFERENCES}

[1] NIH Consensus Development Panel. Osteoporosis prevention, diagnosis, and therapy. JAMA 2001, 285: 785-95.

[2] Consensus development conference. diagnosis, prophylaxis, and treatment of osteoporosis. Am J Med 2003; 94:646-50.

[3] Kelly PJ, Pocock NA, Sambrook PN, Eisman JA. Age and menopauserelated changes in indices of bone turnover. J Clin Endocrinol Metab 1989, 69: 1160-5.

[4] Yasumura S, Aloia JF, Gundberg CM, et al. Serum osteocalcin and total body calcium in normal pre- and postmenopausal women and postmenopausal osteoporotic patients. J Clin Endocrinol Metab 1987; 64: 681-5.

[5] Kamimura M, Uchiyama S, Takahara K, Hashidate H, Kawaguchi A, Nakagawa H. Urinary Excretion of Type I Collagen Cross-linked NTelopeptide and Serum Bone-specific alkaline phosphatase. Age and back pain related changes in elderly women. J Bone Miner Metab 2005; 23: 495-500.

[6] Hosking DJ. Changes in serum alkaline phosphatase after femoral fractures. JBJS $\mathrm{Br}$ 1978; 60: 61-5.

[7] Obrant KJ, Merle B, Bejui J, Delmas PD. Serum bone-gla protein after fracture. Clin Orthop Related Res 1990; 258: 300-3

[8] Ohishi T, Takahashi M, Kushida K, et al. Changes of biochemical markers during fracture healing. Arch Orthop Trauma Surg 1998; 118: 126-30.

[9] Ingel BM, Hay SM, Bottjer HM, Eastell R. Changes in bone mass and bone turnover following distal forearm fracture. Osteoporos Int 1999; 10: 399-407.
[10] Obrant K, Ivaska K, Gerdhem P, Alatalo S, Pettersson K, Väänänen H. Biochemical markers of bone turnover are influenced by recently sustained fracture. Bone 2005; 36: 786-92.

[11] Yu Yahiro JA, Michael RH, Dubin NH, et al. Serum and urine markers of bone metabolism during the year after hip fracture. J Am Geriatr Soc 2001; 49: 877-83.

[12] Akesson K, Kakonen SM, Josefsson PO, Karlsson MK, Obrant KJ, Pettersson K. Fracture-induced changes in bone turnover: a potential confounder in the use of biochemical markers in osteoporosis. J Bone Miner Metab 2005; 23: 30-5.

[13] Nakagawa H, Kamimura M, Uchiyama $S$, et al. Changes in total alkaline phosphatase (ALP) level after hip fracture: comparison between femoral neck and trochanter fracture. J Orthop Sci 2006; 11: 135-59.

[14] Takahara K, Kamimura M, Nakagawa H, Uchiyama S. Changes in biochemical markers of bone in patients with insufficiency fractures. J Bone Miner Metab 2004; 22: 618-25.

[15] Takahara K, Kamimura M, Hashidate H, Uchiyama S, Nakagawa H. Change of cross-linked telopeptide of type I collagen (I CTP) in patients with bone fragility fractures. J Orthop Sci 2007; 12: 219-26.

[16] Lips P, van Ginkel FC, Netelenbos JC, Weisinga A, van der Vijgh WJF. Lower mobility and markers of bone resorption in the elderly. Bone Miner 1990; 9: 49-57.

[17] Pedersen BJ, Schlemer A, Hassager C, Christiansen C. Changes in the carboxyl-terminal propeptide of type I procollagen and other markers of bone formation upon five days of bed rest. Bone 1995; 17: 91-5.

[18] Gregg EW, Cauley JA, Seeley DG, Ensrud KE, Bauer DC. Physical activity and osteoporotic fracture risk in older women. Ann Intern Med 1998; 129: 81-8.

[19] Theiler R, Stähelin HB, Kränzlin M, Tyndall A, Bischoff HA. High turnover in the elderly. Arch Phys Med Rehabil 1999; 80: 485-9.

[20] Inoue M, Tanaka H, Moriwake T, Oka M, Sekiguchi C, Seino Y. Altered biochemical markers of bone turnover in humans during 120 days of bed rest. Bone 2000; 26: 281-6.

[21] Takahara K, Kamimura M, Nakagawa H, Hashidate H, Uchiyama S. Radiographic evaluation of vertebral fractures in osteoporotic patients. J Clinic Neurosci 2007; 14: 122-6.

[22] Fnisen V. Osteoporosis and back pain among the elderly. Acta Med Scand 1988; 223: 443-9.

[23] Cook GJ, Hannaford E, See M, Clarke SE, Fogelman I: The value of bone scintigraphy in the evaluation of osteoporotic patients with back pain. Scand J Reumatol 2002; 31: 245-8.

[24] Lee YL, Yip KM. The osteoporotic spine. Clin Orthop Relat Res 1996; 323: 91-7.

[25] Vogt TM, Ross PD, Palermo L, et al. Vertebral fracture prevalence among women screened for the Fracture Intervention Trial and a simple clinical tool to screen for undiagnosed vertebral fractures. Fracture Intervention Trial Research Group. Mayo Clin Proc 2000; 75: 888-96.

[26] Hanson DA, Weis MA, Bollen AM, Maslan SL, Singer FR, Eyre DR. A specific immunoassay for monitoring human bone resorption: quantitation of type 1 collagen cross-linked $\mathrm{N}$-telopeptides in urine. $\mathrm{J}$ Bone Miner Res 1997; 7: 1251-8.

[27] Ikegami S, Kamimura M, Nakagawa H, et al. Comparison in bone turnover markers during early healing of femoral neck fracture and trochanteric fracture in elderly patients. Orthop Rev 2009; 1:e21.

[28] Ross PD, Fujiwara S, Huang C, et al. Vertebral fracture prevalence in women in Hiroshima compared to Caucasians or Japanese in the US. Int J Epidemiol 1995; 24: 1171-7.

[29] Riggs BL, Melyon LJ III. The worldwide problem of osteoporosis: insights afforded by epidemiology. Bone 1995; 17: S505-S511.

[30] Garnero P, Sornay-Rendu E, Chaustrat B, Delmas PD. Biochemical markers of bone turnover, endogenous hormones and the risk of fractures in postmenopausal women. J Bone Miner Res 2000; 15: 152636.

[31] Lindsay R, Silverman SL, Cooper C, et al. Risk of new vertebral fracture in the year following a fracture. JAMA 2001; 285: 320-3. 